\title{
THE ERROR IN NUMERICAL INTEGRATION OF ANALYTIC FUNCTIONS*
}

\author{
BY \\ SEYMOUR HABER \\ National Bureau of Standards
}

1. Introduction. In the numerical calculation of the integral

$$
I(f)=\int_{a}^{b} f(x) d x
$$

by means of a linear quadrature formula

$$
Q(f)=\sum_{r=1}^{n} a_{i} f\left(x_{i}\right) ; \quad a_{i} \text { real, } \quad x_{i} \in[a, b], \quad i=1, \cdots, n,
$$

when $f$ belongs to a certain Banach space $B$, it may happen that the error functional $E^{Q}$-defined by $E^{Q}(f)=I(f)-Q(f)$-is bounded; and then an upper bound for the quadrature error may be given in the form

$$
|I(f)-Q(f)| \leq\left\|E^{Q}\right\| \cdot\|f\|
$$

(where "\| ||" stands for the norm in $B$ or in $B^{*}$, as appropriate).

Davis [1] introduced the idea of considering the bound (3) in the case that $B$ is the Hardy space $H^{2}$ of functions analytic in the unit disk $|z|<1$ and having finite norm defined by

$$
\|f\|^{2}=2 \pi \sum_{n=0}^{\infty}\left|a_{n}\right|^{2}
$$

(where $f(z)=\sum_{n=0}^{\infty} a_{n} z^{n}$ ). For such $f$, the radial limit, $\lim _{r \rightarrow 1-} f\left(r e^{i \theta}\right)$, exists for almost all $\theta$, and may be taken to define $f$ on the unit circle $C_{1} ; f$ is then in $L^{2}$ on the unit circle, and in fact

$$
\|f\|^{2}=\int_{C_{2}}|f(z)|^{2} d s
$$

In this case $B$ is a Hilbert space, and Davis considered integration over various intervals $[a, b]$ with $-1<a<b<1$. It seems more convenient, however, to fix the integration interval as $[-1,1]$, and to consider functions analytic inside the circle $C_{R}:|z|=R$, for values of $R$ greater than one. I shall denote this space " $H^{2}\left(C_{R}\right)$ "; if $f(z)=\sum_{n=0}^{\infty} a_{n} z^{n}$, the norm is now defined by

$$
\|f\|^{2}=2 \pi R \sum_{n=0}^{\infty}\left|a_{n}\right|^{2} R^{2 n}=\int_{C_{B}}|f(z)|^{2} d s
$$

* Received September 24, 1970. 
or, equivalently, the inner product is defined by

$$
(f, g)=\int_{C_{R}} f(z) \overline{g(z)} d s .
$$

Davis pointed out that an advantage of the error bound (3) over the classical error bounds, in this case, is that it does not involve derivatives of the integrand $f$ and so may be much easier to use.

A little while later, Davis and Rabinowitz [2] made use of the spaces $L^{2}\left(\varepsilon_{\rho}\right)$ for the same purpose. $\mathcal{E}_{\rho}$, for $\rho>1$, is the ellipse

$$
x^{2} / a^{2}+y^{2} / b^{2}=1,
$$

where

$$
a=\frac{1}{2}\left(\rho^{1 / 2}+\rho^{-1 / 2}\right), \quad b=\frac{1}{2}\left(\rho^{1 / 2}-\rho^{-1 / 2}\right) .
$$

All these ellipses have their foci at \pm 1 on the $x$ axis; if $\rho^{\prime}<\rho$ then $\varepsilon_{\rho}$, is inside $\varepsilon_{\rho}$, and as $\rho$ decreases to $1, \varepsilon_{\rho}$ shrinks down to the interval $[-1,1]$ on the $x$ axis. The advantage of this family of regions is that any function that is analytic on the segment $[-1,1]$ is necessarily analytic inside $\varepsilon_{\rho}$ for some $\rho>1$, so that the resulting analysis applies to the numerical integration of any function analytic on the (closed) interval of integration. $L^{2}\left(\varepsilon_{\rho}\right)$ consists of all functions $f$ analytic in the interior $D_{\rho}$ of $\varepsilon_{\rho}$ and such that

$$
\iint_{D_{\rho}}|f(z)|^{2} d x d y<\infty ;
$$

it is a Hilbert space with inner product

$$
(f, g)=\iint_{D_{\rho}} f(z) \overline{g(z)} d x d y .
$$

In this paper $L^{2}\left(\varepsilon_{\rho}\right)$, like $H^{2}\left(C_{R}\right)$, will always be used in connection with integration over $[-1,1]$.

Davis and Rabinowitz, Hämmerlin, and others have given a number of evaluations and bounds for the error norms $\left\|E^{Q}\right\|$, for the space $H^{2}\left(C_{R}\right)$ and $L^{2}\left(\varepsilon_{\rho}\right)$, and various quadrature formulas $Q$ [1]-[8].

A few years ago Wilf [11] considered the space $H^{2}\left(C_{1}\right)$ in connection with integration over the interval $[0,1]$. This case differs greatly from those mentioned above, in that the closed interval of integration is not contained in the region of analyticity of the functions-integrands are admitted which are not analytic at one endpoint. I shall refer to this case by the symbol " $H^{2}\left(C_{1} ; 0,1\right)$ ". The study of integration over $[-1,1]$ of functions in $H^{2}\left(C_{1}\right)$ is essentially the same [12].

In all cases a problem of obvious interest is the determination, for each specific number $n$ of quadrature abscissas, of that quadrature formula $Q$ for which $\left\|E^{Q}\right\|$ is as small as possible. There is a unique $Q$ with this property, whenever the function space $B$ is a Hilbert space, or, more generally, when $B^{*}$ is strictly convex. Such a formula is called a "minimum-norm" formula (relative to the specified number $n$ ); we shall denote it by " $Q_{n}^{\mathrm{MN}}$ " and its error functional by " $E_{n}^{\mathrm{MN}}$ ". It is defined by

$$
\left|E_{n}^{\mathrm{MN}}\right|=\inf _{\substack{a_{1}, a_{a}, \ldots, a_{n} \\ a \leq x_{1}, x_{n}, \ldots, x_{n} \leq b}}\left\|E^{Q}\right\|
$$


where $Q$ is given by (2). The explicit determination of the $a_{i}$ and $x_{i}$ of such formulas is quite difficult and has been done only in very few cases [8], [10]. Valentin [13] proved that for the spaces $H^{2}\left(C_{R}\right)$ the abscissas and coefficients of $Q_{n}^{\mathrm{MN}}$, for any fixed $n$, approach those of the $n$-point Gauss-Legendre quadrature formulas as $R \rightarrow \infty$; proof of the same fact for $L^{2}\left(\varepsilon_{\rho}\right)$, as $\rho \rightarrow \infty$, can be found in [14]. (For information about the Gaussian quadrature formulas see, e.g., [16, chapter 8$]$.)

2. Bounds on $\left\|E_{n}^{\mathrm{MN}}\right\|$. In some recent papers [11], [14], [15] bounds on $\left\|E_{n}^{\mathrm{MN}}\right\|$, and on the asymptotic size of $\left\|E_{n}^{\mathrm{MN}}\right\|$ as $n \rightarrow \infty$, have been derived by the technique of calculation of the norm of the error functional of some convenient $n$-point quadrature formula $Q_{n}$, using the inequality

$$
\left\|E_{n}^{\mathrm{MN}}\right\| \leq\left\|E^{Q_{n}}\right\|
$$

(which follows from (6)). Stetter [15] used the repeated midpoint (or "Euler's") rule,

$$
M_{n}(f)=\frac{1}{n} \sum_{r=1}^{n} f\left(\frac{n-2 r+1}{n}\right),
$$

for the spaces $H^{2}\left(C_{R}\right)$ and $L^{2}\left(\varepsilon_{p}\right)$, and showed that in both cases

$$
\left\|E_{n}^{\mathrm{MN}}\right\|=O\left(n^{-2}\right)
$$

for any $R, \rho>1$. Barnhill [14] found a bound on $\left\|E^{G_{n}}\right\|$ for $L^{2}\left(\varepsilon_{\rho}\right)$, where $G_{n}$ is the $n$-point Gauss-Legendre quadrature formula, and applied it to $\left\|E_{n}^{\mathrm{MN}}\right\|$. For large values of $\rho$ his bound approaches zero exponentially as $n \rightarrow \infty$ (i.e., it is $O\left(c^{-n}\right)$, for some $c>1$ ) and thus improves on (9); but for $\rho<e^{2}$ it approaches infinity as $n$ increases.

For the case $H^{2}\left(C_{1} ; 0,1\right)$, Wilf showed that

$$
\left\|E_{n}^{\mathrm{MN}}\right\|=O(\log n / n)^{1 / 2} \text {. }
$$

This stands in considerable contrast to what can be shown in the other cases. I give a slightly better result below, but I do not think any great improvement is possible.

TheOREM 1. Let $D$ be a simply-connected open set in the complex plane that contains the closed interval $[a, b]=[-1,1]$ on the $x$-axis. Let $S$ be a Hilbert space of functions analytic on $D$ with the following property: If $D_{c}$ is any compact subset of $D$, there is a positive number $M=M\left(D_{c}\right)$ such that

$$
\max _{z \in D_{c}}|f(z)| \leq M\|f\| \text { for all } f \in S .
$$

Then if $Q$ is of the form (2), the functional $E^{\mathcal{Q}}$ is bounded; and if

$$
\rho=\rho(D)=\sup \left\{\rho^{\prime}: \varepsilon_{\rho^{\prime}} \subset D\right\},
$$

and $\epsilon$ is any number between 0 and $\rho-1$, there is a positive number $A=A(\epsilon)$ such that

$$
\left\|E_{n}^{\mathrm{MN}}\right\| \leq\left\|E^{G_{n}}\right\| \leq A(\rho-\epsilon)^{-n} \text {. }
$$

(This theorem can be applied to integration over any finite interval $[a, b]$, by a linear change of variables. The interval $[-1,1]$ was used so as not to complicate the forms of (12) and (13).)

Proof. Since the interval $[-1,1]$ is a compact subset of $D$, the assumption (11) immediately implies the boundedness of the functionals $I$ and $Q$, and so of $E^{Q}$. The rest 
of the theorem follows from a classical result on polynomial approximation: Any $f \in S$ is analytic on and inside $\varepsilon_{\rho-e}$; it then follows from the proof of Theorem 7, p. 76, of [17] ${ }^{(1)}$ that there is a constant $A_{1}=A_{1}(\rho, \epsilon)$ such that for any positive integer $m$ there exists a polynomial $P_{m}(z)$, of degree $m$, such that

$$
\max _{z \in[-1,1]}\left|f(z)-P_{m}(z)\right| \leq A_{1}(\rho-\epsilon)^{-m / 2} \mu,
$$

where $\mu$ is the maximum of the absolute value of $f$ on $\varepsilon_{p-e}$. By (11), there is an $M$ such that $\mu \leq M\|f\|$, so that we can write

$$
\max _{z \in[-1,1]}\left|f(z)-P_{m}(z)\right| \leq A_{2}(\rho-\epsilon)^{-m / 2}\|f\| .
$$

Now $G_{n}$ integrates polynomials of degree $2 n-1$ exactly, and the sum of the absolute values of the coefficients in $G_{n}$ is 2 ; so that

$$
\begin{aligned}
\left|E^{G_{n}}(f)\right| & =\left|E^{G_{n}}\left(f-P_{2 n-1}\right)\right| \\
& \leq\left|I\left(f-P_{2 n-1}\right)\right|+\left|G_{n}\left(f-P_{2 n-1}\right)\right| \\
& \leq 4 A_{2}(\rho-\epsilon)^{-(2 n-1) / 2}|| f|| .
\end{aligned}
$$

(13) now follows, with $A=4 A_{2} \rho^{1 / 2}$.

It is easy to see that the spaces $L^{2}\left(\varepsilon_{\rho}\right)$ and $H^{2}\left(C_{R}\right)$ satisfy the hypothesis (11). If $f \in L^{2}\left(\varepsilon_{\rho}\right)$, let $z_{0}$ be a point such that

$$
\left|f\left(z_{0}\right)\right| \geq \max _{z \in D_{c}}|f(z)| .
$$

There is a neighborhood of $z_{0}$-of area $\alpha$, say-in which $|f(z)| \geq \frac{1}{2}\left|f\left(z_{0}\right)\right|$. Then

$$
\|f\|^{2} \geq \frac{\alpha}{4}\left|f\left(z_{0}\right)\right|^{2}
$$

A similar argument, using the maximum modulus theorem to locate $z_{0}$ on $C_{R}$, works for $H^{2}\left(C_{R}\right)$.

$H^{2}\left(C_{R}\right)$ has the orthonormal basis

$$
\varphi_{n}(z)=(2 \pi R)^{-1 / 2}(z / R)^{n}, \quad n=0,1,2, \cdots ;
$$

$L^{2}\left(\varepsilon_{\rho}\right)$ has the orthonormal basis

$$
\varphi_{n}(z)=\left(\frac{2(n+1)}{\pi}\right)^{1 / 2}\left(\rho^{n+1}+\rho^{-n-1}\right)^{-1 / 2} U_{n}(z), \quad n=0,1,2, \cdots,
$$

where $U_{n}$ is the $n$th degree Chebyshev polynomial of the second kind (see, e.g., [2]). In each case, the functions in the orthonormal basis are real when $z$ is real. For such spaces, Theorem 1 can be given a more specific form. Define the "kernel function" $K$ (using a standard notation) by

$$
K(z, \bar{w})=\sum_{r=0}^{\infty} \varphi_{r}(z) \overline{\varphi_{r}(w)} .
$$

(1) Note that in describing ellipses the " $\rho$ " of [17] is the square root of the $\rho$ of the present paper. There is a slight misstatement in the proof in [17]: the function $g$ used there is not necessarily analytic on the ring $R$, as stated, but is only continuous on $R$ and analytic in the subrings $\rho^{-1} \leq|z|<1$ and $1<|z| \leq \rho$. The rest of the proof is not affected. 
$K$ is real for real $z$ and $w$; and we have

$$
\begin{aligned}
\left\|E^{0}\right\|^{2}=\sum_{r=0}^{\infty}\left|E^{0}\left(\varphi_{r}\right)\right|^{2} \\
\quad=\sum_{r=0}^{\infty} E_{(z)}^{Q}\left(\varphi_{r}(z)\right) E_{(w)}^{Q} \overline{\left(\varphi_{r}(w)\right)} \\
=E_{(z)}^{0} E_{(w)}^{0}(K(z, \bar{w})) .
\end{aligned}
$$

(Here the subscript " $(z)$ " or " $(w)$ " indicates with respect to which variable the functional is operating.) For $0<\epsilon<\rho-1$, let $M$. be the maximum of $|K(z, \bar{w})|$ for $z, w \in \mathcal{E}_{\rho-\text {.. }}$ By Theorem 4 of [19], it follows that

$$
\left|E_{(w)}^{G n}(K(z, w))\right|<\frac{32}{\pi} M_{\epsilon}(\rho-\epsilon)^{-n}
$$

for all $z$ inside $\varepsilon_{\rho-\_} . E_{(w)}^{G_{*}}(K(z, \bar{w}))$ is itself analytic in $D$, and real for real $z$; applying the same theorem again, we have

$$
\left\|E^{G_{n}}\right\|^{2}<\left[\frac{32}{\pi}(\rho-\epsilon)^{-n}\right]^{2} M .
$$

and

$$
\left\|E_{n}^{\mathrm{MN}}\right\| \leq\left\|E^{G_{n}}\right\| \leq \frac{32}{\pi} M_{\bullet}^{1 / 2}(\rho-\epsilon)^{-n}
$$

For $H^{2}\left(C_{B}\right)$, the $\rho$ of $(12)$ is equal to $2 R^{2}-1+2 R\left(R^{2}-1\right)^{1 / 2}$, and

$$
K(z, \bar{w})=\frac{R}{2 \pi\left(R^{2}-z \bar{w}\right)},
$$

$K$ obviously attains its maximum on $\varepsilon_{\rho-\epsilon}$ at

$$
z=\bar{w}=\max \left\{\operatorname{Re}(z): z \in \mathcal{E}_{\rho-\ell}\right\}=\frac{(\rho-\epsilon)^{1 / 2}+(\rho-\epsilon)^{-1 / 2}}{2} .
$$

Since $R=\left(\rho^{1 / 2}+\rho^{-1 / 2}\right) / 2$, we have that

$$
\begin{aligned}
M_{\epsilon} & =\frac{\rho^{1 / 2}+\rho^{-1 / 2}}{\pi}\left(\epsilon-\frac{\epsilon}{\rho(\rho-\epsilon)}\right)^{-1} \\
& \leq \frac{\rho^{1 / 2}+\rho^{-1 / 2}}{\pi}\left(\epsilon-\frac{\epsilon}{\rho}\right)^{-1}=\frac{\epsilon^{-1} \rho^{-1 / 2}}{\pi} \frac{\rho^{1 / 2}+\rho^{-1 / 2}}{\rho^{1 / 2}-\rho^{-1 / 2}} .
\end{aligned}
$$

Thus

$$
\left\|E^{G n}\right\| \leq 32 \pi^{-3 / 2} \rho^{1 / 4} \epsilon^{-1 / 2}\left(\frac{\rho^{1 / 2}+\rho^{-1 / 2}}{\rho^{1 / 2}-\rho^{-1 / 2}}\right)^{1 / 2}(\rho-\epsilon)^{-n}
$$

This last expression is least when $\epsilon=\rho /(2 n+1)$; if $\rho>1+1 /(2 n)$ this is an admissible value of $\epsilon$. Since

$$
\left(\rho-\frac{\rho}{2 n+1}\right)^{-n}=\rho^{-n}\left(1+\frac{1}{2 n}\right)^{n}<e^{1 / 2} \rho^{-n}
$$


that value of $\epsilon$ gives us the bound

$$
\left\|E^{G_{n}}\right\| \leq B_{1}(2 n+1)^{1 / 2} \rho^{-n}
$$

where

$$
B_{1}=32 e^{1 / 2} \pi^{-3 / 2} \rho^{-1 / 4}\left(\frac{\rho^{1 / 2}+\rho^{-1 / 2}}{\rho^{1 / 2}-\rho^{-1 / 2}}\right)^{1 / 2} .
$$

For $L^{2}\left(\varepsilon_{\rho}\right)$, the $\rho$ of $(12)$ is just $\rho$, and

$$
K(z, \bar{w})=\frac{4}{\pi} \sum_{r=0}^{\infty}(r+1) \frac{U_{r}(z) \overline{U_{r}(w)}}{\rho^{r+1}-\rho^{-r-1}} .
$$

For $z \in[-1,1],\left|U_{r}(z)\right| \leq r+1$, since then

$$
U_{r}(z)=\frac{\sin [(r+1) \arccos z]}{\sin [\arccos z]} ;
$$

and it follows, by a classical theorem of Berstein [17, page 42] that

$$
\left|U_{r}(z)\right| \leq(r+1)(\rho-\epsilon)^{r / 2}
$$

throughout $\varepsilon_{p-\mathrm{e}}$. Thus

$$
M_{.} \leq \frac{4}{\pi} \sum_{r=0}^{\infty}(r+1)^{3} \frac{(\rho-\epsilon)^{r}}{\rho^{r+1}-\rho^{-r-1}}<\frac{4}{\pi\left(\rho-\rho^{-1}\right)} \sum_{r=0}^{\infty}(r+1)^{3}(1-\epsilon / \rho)^{r} .
$$

Since

$$
\sum_{r=0}^{\infty}(r+1)^{3} x^{r}=\frac{1+4 x+x^{2}}{(1-x)^{4}}<\frac{6}{(1-x)^{4}},
$$

for $|x|<1$, we have

$$
M_{e}<\frac{24}{\pi\left(\rho-\rho^{-1}\right)} \rho^{4} \epsilon^{-4} .
$$

We can then minimize with respect to $\epsilon$, as was done above; and in sum we obtain:

ThEOREM 2: For $H^{2}\left(C_{R}\right)$,

$$
\left\|E_{n}^{\mathrm{MN}}\right\| \leq\left\|E^{G_{n}}\right\| \leq B_{1}(2 n+1)^{1 / 2} \rho^{-n}
$$

if $\rho>1+1 /(2 n)$, where $\rho=2 R^{2}-1+2 R\left(R^{2}-1\right)^{172}$, and

$$
B_{1}=32 e^{1 / 2} \pi^{-3 / 2} \rho^{-1 / 4}\left(\frac{\rho^{1 / 2}+\rho^{-1 / 2}}{\rho^{1 / 2}-\rho^{-1 / 2}}\right)^{1 / 2} .
$$

For $L^{2}\left(\varepsilon_{p}\right)$,

$$
\left\|E_{n}^{\mathrm{MN}}\right\| \leq\left\|E^{G n}\right\| \leq B_{2}(n+2)^{2} \rho^{-n}
$$

if $\rho>1+2 / n$, where

$$
B_{2}=\frac{2^{8} 6^{1 / 2} e^{2}}{\pi^{3 / 2}\left(\rho-\rho^{-1}\right)^{1 / 2}}
$$

3. The case $H^{2}\left(C_{1} ; 0,1\right)$. If $0 \leq X<1$, let $P_{x}$ denote the "point functional" defined by

$$
P_{z}(f)=f(x), \quad f \in H^{2}\left(C_{1}\right)
$$


Then

$$
P_{x}(f)=\frac{1}{2 \pi i} \int_{C_{2}} \frac{f(z)}{z-x} d z=\frac{1}{2 \pi} \int_{0}^{2 \pi} \frac{e^{i \theta} f\left(e^{i \theta}\right)}{e^{i \theta}-x} d \theta .^{(2)}
$$

So, applying the Schwartz inequality,

$$
\left|P_{x}(f)\right| \leq \frac{1}{2 \pi}\left(\int_{0}^{2 x} \frac{d \theta}{\left|e^{i \theta}-x\right|^{2}}\right)^{1 / 2} \cdot\|f\|
$$

and it follows that $P_{x}$ is bounded. Thus quadrature functionals $Q$ of the form (2) (with $[a, b]=[0,1])$ are bounded, if we exclude the use of the point $x=1$ as an abscissa in $Q$. (This must be done in any case, if $Q$ is to be used to integrate arbitrary functions in $H^{2}\left(C_{1}\right)$, as these are not all well defined at 1.) To show that $E^{Q}$ is bounded, we show that $I$ is:

$$
I(f)=\int_{0}^{1} f(t) d t=\lim _{x \rightarrow 1-} \int_{0}^{x} f(t) d t
$$

and

$$
\begin{aligned}
\int_{0}^{x} f(t) d t & =\frac{1}{2 \pi i} \int_{0}^{x} \int_{C_{1}} \frac{f(z)}{z-t} d z d t \\
& =\frac{1}{2 \pi} \int_{0}^{2 \pi} e^{i \theta} f\left(e^{i \theta}\right) \int_{0}^{x} \frac{d t}{e^{i \theta}-t} d \theta
\end{aligned}
$$

Therefore

$$
\left|\int_{0}^{x} f(t) d t\right| \leq \frac{1}{2 \pi} \cdot\|f\| \cdot\left(\int_{0}^{2 \pi}\left|\log \frac{e^{i \theta}}{e^{i \theta}-x}\right|^{2} d \theta\right)^{1 / 2} .
$$

The last integrand is bounded except in the neighborhood of 0 and of $2 \pi$, so we can write (letting " $C$ " denote a generic constant, different in different occurrences, but in each case independent of $x$ )

$$
\begin{aligned}
\int_{0}^{2 \pi}\left|\log \frac{e^{i \theta}}{e^{i \theta}-x}\right|^{2} d \theta & \leq C+C \int_{0}^{\pi / 2}\left|\log \left(e^{i \theta}-x\right)\right|^{2} d \theta \\
& \leq C+C \int_{0}^{\pi / 2}|\log \sin \theta|^{2} d \theta \\
& \leq C+C \int_{0}^{\pi / 2} \log ^{2} \theta d \theta \leq C^{*}
\end{aligned}
$$

Thus

$$
\left|\int_{0}^{x} f(t) d t\right| \leq \frac{C^{*}}{2 \pi}\|f\|
$$

for all $x$, and it follows that the functional $I$ is also bounded.

A complete orthonormal sequence for $H^{2}\left(C_{1}\right)$ is given by (16), with $R=1$, and $K(z, \bar{w})=1 / 2 \pi(1-z \bar{w})$. If we set

$$
F(z)=I_{(w)}(K(z, \bar{w}))=\frac{1}{2 \pi z} \log \frac{1}{1-z}
$$

(2) For the validity of the Cauchy integral formula for functions in $H^{2}$ see, e.g., [21, p. 332]. 
then $F(z)=\sum_{r=0}^{\infty} I\left(\varphi_{r}\right) \varphi_{r}(z)$. Noting that

$$
I\left(\varphi_{r}\right)=I\left(\bar{\varphi}_{r}\right)=\overline{I\left(\varphi_{r}\right)}
$$

and similarly for $Q$, we may write

$$
\begin{aligned}
\left\|E^{Q}\right\|^{2} & =\sum_{r=0}^{\infty}\left|E^{\Theta}\left(\varphi_{r}\right)\right|^{2}=\sum_{r=0}^{\infty}\left(I\left(\varphi_{r}\right)-Q\left(\varphi_{r}\right)\right)^{2} \\
& =\sum_{r=0}^{\infty}\left[I\left(I\left(\varphi_{r}\right) \varphi_{r}\right)-Q\left(I\left(\varphi_{r}\right) \varphi_{r}\right)\right]-\sum_{r=0}^{\infty} Q\left(\varphi_{r}\right)\left[I\left(\varphi_{r}\right)-Q\left(\varphi_{r}\right)\right] \\
& =E^{Q}(F)-Q_{(z)} E_{(w)}^{Q}(K) .
\end{aligned}
$$

Here I shall take, for the comparison formula $Q$, Euler's rule

$$
M_{n}(f)=\frac{1}{n} \sum_{r=0}^{n} f\left(\frac{2 r-1}{2 n}\right) .
$$

To estimate $E^{\mathcal{S n}_{n}}$ I shall use the Peano remainder form (see, e.g., [20, p. 108])

$$
E^{M_{n}}(f)=\sum_{r=1}^{n} \int_{r-1 / n}^{r / n} f^{\prime \prime}(t)\left[\frac{(1-t)^{2}}{2}-\frac{1}{n} \sum^{*}\left(\frac{2 k-1}{2 n}-t\right)\right] d t \equiv \sum_{r=1}^{n} e_{r}(f)
$$

where the sum $\Sigma^{*}$ is over those values of $k$ between 1 and $n$ for which $(2 k-1) / 2 n \geq t$. (The statement of the Peano theorem in [20] assumes that $f^{(n+1)}$-in the present case $f^{\prime \prime}$-is continuous on the closed integration interval $[a, b]$. However, the usual proof is valid whenever $f^{(n+1)}$ is continuous on $(a, b)$ and $\int_{a}^{b}(b-t)^{n+1} f^{(n+1)}(t) d t$ is finite. $F$ and $K$ satisfy these conditions.)

Looking first at $e_{n}$, we have

$$
\begin{aligned}
e_{n}\left(F^{\prime}\right) & =\int_{1-1 / n}^{1-1 / 2 n} F^{\prime \prime}(t)\left[\frac{(1-t)^{2}}{2}-\frac{1}{n}\left(1-t-\frac{1}{2 n}\right)\right] d t+\int_{1-1 / 2 n}^{1} F^{\prime \prime}(t)\left[\frac{(1-t)^{2}}{2}\right] d t \\
& =\int_{1-1 / n}^{1} \frac{(1-t)^{2}}{2} F^{\prime \prime}(t) d t-\frac{1}{n} \int_{1-1 / n}^{1-1 / 2 n}(1-t) F^{\prime \prime}(t) d t+\frac{1}{2 n^{2}} \int_{1-1 / n}^{1-1 / 2 n} F^{\prime \prime}(t) d t .
\end{aligned}
$$

Substituting

$$
F^{\prime \prime}(t)=\frac{1}{2 \pi}\left[\frac{1}{t(1-t)^{2}}-\frac{2}{t^{2}(1-t)}+\frac{2}{t^{3}} \log \frac{1}{1-t}\right]
$$

in the last 3 integrals and seeing that each of the last 2 terms in (31) contributes only $O\left(n^{-2}\right)$ to each of the 3 terms in (30), we obtain

$$
\begin{aligned}
2 \pi e_{n}(F) & =\frac{1}{2} \int_{1-1 / n}^{1} \frac{d t}{t}-\frac{1}{n} \int_{1-1 / n}^{1-1 / 2 n} \frac{d t}{t(1-t)}+\frac{1}{2 n^{2}} \int_{1-1 / n}^{1-1 / 2 n} \frac{d t}{t(1-t)^{2}}+O\left(\frac{1}{n^{2}}\right) \\
& =\frac{1}{2} \int_{1-1 / n}^{1} d t-\frac{1}{n} \int_{1-1 / n}^{1-1 / 2 n} \frac{d t}{1-t}+\frac{1}{2 n^{2}} \int_{1-1 / n}^{1-1 / 2 n} \frac{d t}{(1-t)^{2}}+O\left(\frac{1}{n^{2}}\right) \\
& =\frac{1-\log 2}{n}+O\left(\frac{1}{n^{2}}\right) .
\end{aligned}
$$


For $r<n$,

$$
\begin{aligned}
e_{r}(F)=\int_{(r-1) / n}^{(r-1 / 2) / n} F^{\prime \prime}(t) & {\left[\frac{(1-t)^{2}}{2}-\frac{1}{n} \sum_{k=r}^{n}\left(\frac{2 k-1}{2 n}-t\right)\right] d t } \\
& +\int_{(r-1 / 2) / n}^{r / n} F^{\prime \prime}(t)\left[\frac{(1-t)^{2}}{2}-\frac{1}{n} \sum_{k=r+1}^{n}\left(\frac{2 k-1}{2 n}-t\right)\right] d t .
\end{aligned}
$$

The quantities in brackets in (29), and so in (33), are nonnegative for all $t$; in the case $N=1$ this can be determined by inspection, and it follows for all $n$ by the corollary on p. 111 of [20]. Therefore

$$
\begin{aligned}
e_{r}\left(F^{\prime}\right)= & F^{\prime \prime}(\xi) \int_{(r-1) / n}^{(r-1 / 2) / n}\left[\frac{(1-t)^{2}}{2}-\frac{1}{n} \sum_{k=r}^{n}\left(\frac{2 k-1}{2 n}-t\right)\right] d t \\
& \quad+F^{\prime \prime}\left(\xi_{2}\right) \int_{(r-1 / 2) / n}^{r / n}\left[\frac{(1-t)^{2}}{2}-\frac{1}{n} \sum_{k=r+1}^{n}\left(\frac{2 k-1}{2 n}-t\right)\right] d t \\
= & F^{\prime \prime}\left(\xi_{3}\right) \int_{r-1 / n}^{r / n}\left[\frac{(1-t)^{2}}{2}-\frac{1}{n} \sum^{*}\left(\frac{2 k-1}{2 n}-t\right)\right] d t \\
= & \frac{F^{\prime \prime}\left(\xi_{3}\right)}{24 n^{3}}
\end{aligned}
$$

where $\xi_{1}, \xi_{2}$, and $\xi_{3}$ are numbers in $((r-1) / n, r / n)$. Now

$$
F(t)=\frac{1}{2 \pi}\left(1+\frac{t}{2}+\frac{t^{2}}{3}+\cdots\right)
$$

so that $F^{\prime \prime}(t)$ is continuous at $t=0$, and increasing in $t$. It follows from (31) that

$$
0 \leq F^{\prime \prime}(t) \leq C /(1-t)^{2}
$$

for some constant $C$, and this and (33) imply that

$$
\left|e_{n}(F)\right| \leq C / 24 n(n-r)^{2}, \quad r=1,2, \cdots, n-1 .
$$

Thus

$$
\mid \sum_{r=1}^{n-1} e_{n}\left(F^{\prime}\right) \leq \frac{C}{24 n} \sum_{r=1}^{n-1} \frac{1}{(n-r)^{2}} \leq \frac{C^{\prime}}{n} ;
$$

this, together with (32), implies that $\left|E^{M_{n}}(F)\right|=O(1 / n)$.

To bound the other term on the right side of $(27)$, we note that for $z \in[0,1]$

$$
\begin{aligned}
& E_{(w)}^{M_{n}}(K(z, \bar{w}))=\frac{1}{2 \pi} E_{(t)}^{M_{n}}\left(\frac{1}{1-z t}\right) \\
& \quad=\frac{1}{2 \pi} \int_{0}^{1} \frac{2 z^{2}}{(1-z t)^{3}}\left[\frac{(1-t)^{2}}{2}-\frac{1}{n} \sum *\left(\frac{2 k-1}{2 n}-t\right)\right] d t .
\end{aligned}
$$

Call this last quantity " $g(z)$ ", for convenience. We have seen that the Peano kernel of $M_{n}$-the bracketed quantity in the last integral-is nonnegative and its integral over each interval $(r / n,(r+1) / n)$ is $1 /\left(24 n^{3}\right)$. Furthermore it is continuous, and its derivative, at each point of differentiability, is $<2$ in absolute value. It is therefore $<C / n^{2}$, for some constant $C$, for all $t$ in $[0,1]$. It follows that 


$$
|g(z)|<\frac{C}{n^{2}} \int_{0}^{1} \frac{2 z^{2}}{(1-z t)^{3}} d t=\frac{C}{n^{2}}\left(\frac{z}{(1-z)^{2}}-z\right)<\frac{C}{n^{2}} \frac{1}{(1-z)^{2}}
$$

Then

$$
\begin{aligned}
\left|Q_{(z)} E_{(w)}^{M n}(K(z, \bar{w}))\right|=\left|\frac{1}{n} \sum_{r=1}^{n} g\left(\frac{2 r-1}{2 n}\right)\right|<\frac{C}{n^{3}} \sum_{r=1}^{n}[1 & \left.-\left(\frac{2 r-1}{2 n}\right)\right]^{-2} \\
& <\frac{4 C}{n} \sum_{r=1}^{n}(2 n-2 r+1)^{-2}<\frac{C^{\prime}}{n} .
\end{aligned}
$$

This completes the proof of

TheOREM 3. In the case of $H^{2}\left(C_{1} ; 0,1,\right)$,

$$
\left\|E_{n}^{M N}\right\|^{2} \leq\left\|E^{M M_{n}}\right\|^{2}=O\left(\frac{1}{n}\right)
$$

This looks very weak by comparison with the previous theorems, but I think that it cannot be improved as to order.

\section{REFERENCES}

[1] Philip J. Davis, Errors of numerical approximation for analytic functions, J. Rational Mech. Anal. 2, 303-313 (1953)

[2] Philip J. Davis and Philip Rabinowitz, On the estimation of quadrature errors for analytic functions, MTAC 8, 193-203 (1954)

[3] Günther Hämmerlin, Über ableitungsfreie Schranken für Quadraturfehler, Numer. Math. 5, 226233 (1963)

[4] Günther Hämmerlin, Über ableitungsfreie Schranken für Quadraturfehler. II, Numer. Math. 7, 232-237 (1965)

[5] Günther Hämmerlin, Zur Abschätzung von Quadraturfehlern für analytische Funktionen, Numer. Math. 8, 334-344 (1966)

[6] Y. T. Lo, S. W. Lee and B. Sun, On Davis' method of estimating qudrature errors, Math. Comp .19, 133-138 (1965)

[7] R. E. Barnhill and J. A. Wixom, Quadratures with remainders of minimum norm. I, Math. Comp. 21, 66-75 (1967)

[8] R. E. Barnhill and J. A. Wixom, Quadratures with remainders of minimum norm. II, Math. Comp. 21, 382-387 (1967)

[9] R. E. Barnhill, Optimal quadratures in L2( $\left.\varepsilon_{\rho}\right)$. I, SIAM J. Numer. Anal. 4, 390-397 (1967)

[10] R. E. Barnhill, Optimal quadratures in $L^{2}\left(\varepsilon_{\rho}\right)$. II, SIAM J. Numer. Anal. 4, 534-541 (1967)

[11] H. S. Wilf, Exactness conditions in numerical quadrature, Numer. Math. 6, 315-319 (1964)

[12] H. S. Wilf, Advances in numerical quadrature, Mathematical Methods for Digital Computers, vol. II, Wiley, New York, 1967

[13] R. A. Valentin, Applications of functional analysis to optimal numerical approximation for analytic functions, Doctoral Thesis, Brown University, Providence, R. I., 1965

[14] R. E. Barnhill, Asymptotic properties of minimum norm and optimal quadratures, Numer. Math. 12, 384-393 (1968)

[15] F. Stetter, On best quadrature of analytic functions, Quart. Appl. Math. 27, 270-272 (1969)

[16] F. B. Hildebrand, Introduction to numerical analysis, McGraw-Hill, New York, 1956

[17] G. G. Lorentz, Approximation of functions, Holt, Rinehart and Winston, New York, 1966

[18] N. I. Ahiezer, Lectures in the theory of approximation, OGIZ, Moscow, 1947; English transl., Ungar, New York, 1956

[19] F. Stenger, Bounds on the error of Gauss-type quadratures, Numer. Math. 8, 150-160 (1966)

[20] P. J. Davis and P. Rabinowitz, Numerical integration, Blaisdell, Waltham, Mass., 1967

[21] W. Rudin, Real and complex analysis, McGraw-Hill, New York, 1966 